\title{
Das Cauda-equina-Syndrom des Hundes
}

\author{
Konrad Jurina
}

Die häufigste Ursache für ein Caudaequina-Syndrom bei großen mittelalten bis alten Hunden ist eine degenerative lumbosakrale Stenose. Betroffene Tiere können sich klinisch mit Schmerzen beim Springen, Treppensteigen oder Aufstehen, eingeschränkter Beweglichkeit der Rute oder Lahmheit einer Hintergliedmaße präsentieren. Therapeutisch kann eine konservative medikamentelle Schmerzbehandlung versucht werden, häufig ist aber eine chirurgische Dekompression notwendig.

Unter dem Begriff des Cauda-equinaSyndroms werden zahlreiche unterschiedliche Krankheiten zusammengefasst, die die sogenannte Cauda equina beeinträchtigen. Andere verwendete Bezeichnungen sind lumbosakrale Stenose oder Lumbosakralsyndrom. Es handelt sich um eine relativ häufige Erkrankung großer Hunde, deren Diagnose aber oftmals schwierig sein kann. Die Krankheit ist als neurologisches Problem definiert, äußert sich klinisch aber oftmals eher orthopädisch.

\section{Relevante Anatomie}

Zum Verständnis der klinischen und neurologischen Symptome ist eine Kenntnis der Anatomie der lumbosakralen Region notwendig.

Infolge des unterschiedlichen Längenwachstums im Zuge der Embryonalentwicklung endet das Rückenmark im Wirbelkanal vor dem Ende der Wirbelsäule [5]. Dies ist besonders bei großen Hunden ausgeprägt. Das Ende des Rückenmarks, der Conus medullaris, ist in der Regel in Höhe der kaudalen Hälfte des 6. Lendenwirbels bzw. der kranialen Hälfte des 7. Lendenwirbels gelegen [8]. Bei kleineren Hunden oder Katzen ist er weiter in Richtung des Endes des 7. Lendenwirbels gelegen. Der Durasack endet weiter kaudal und reicht bei vielen Hunden bis in das Os sacrum. Es gibt hier aber zahlreiche Variationen [17].

Die Cauda equina entspringt aus dem Conus medullaris und besteht aus den paarigen Spinalnervenwurzeln bzw. Spinalnerven der Rückenmarkssegmente L6, L7, S1-S3 und Cd1-Cd5. Die Dorsal- und Ventralwurzeln sowie die Spinalnerven liegen innerhalb des Wirbelkanals, laufen über eine Strecke parallel und verlassen den Wirbelkanal durch die Neuroforaminae [6]. Sie erinnern daher an den Schweif eines Pferdes (= Cauda equina).

Das Rückenmark gehört wie das Gehirn zum zentralen Nervensystem, während die Spinalnervenwurzeln, Spinalnerven, neuromuskuläre Endplatte und Muskulatur aus funktionell neurologischer Sicht zum peripheren Nervensystem gezählt werden [30]. Es sind also im Bereich der kaudalen lumbalen bzw. lumbosakralen Wirbelsäule auch Anteile des peripheren Nervensystems gelegen, was wichtig für das Verständnis der klinischen Befunde ist. Im Gegensatz zu anderen Regionen der Wirbelsäule sind die zugehörigen Rückenmarkssegmente nicht auf gleicher Höhe mit den gleichnamigen Wirbelkörpern. So liegen beispielsweise die Rückenmarkssegmente S1-S3 oftmals in Höhe des 6. oder 7. Lendenwirbelkörpers [8].

Im Bereich der lumbosakralen Region der Wirbelsäule werden die Nerven der Cauda equina von folgenden Strukturen umgeben: ventral vom Lig. longitudinale dorsale, dem dorsalen Anulus fibrosus des Intervertebralspalts von L7-S1 und den entsprechenden Wirbelendplatten bzw. -körpern; lateral von den Pedikeln der Wirbelkörper von L7 bzw. S1 mit den Facettengelenken mit synovialen Strukturen und den Neuroforaminae; dorsal

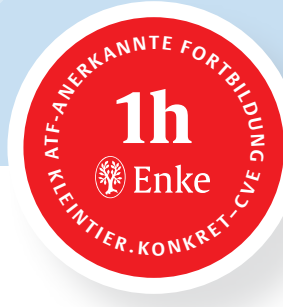

vom Lig. interarcuale (Lig. flavum) und den Laminae der Wirbel L7 und S1.

Stabilisiert wird die lumbosakrale Region weiterhin durch das Lig. interspinosus, das Lig. longitudinale ventrale, die umliegenden Faszien und die Muskulatur [6].

\section{Ursachen}

Die häufigste Ursache des Cauda-equinaSyndroms beim Hund ist eine degenerative lumbosakrale Stenose (DLSS), auf die in diesem Artikel detaillierter eingegangen werden soll.

Weiterhin kommen in Betracht:

- kongenitale lumbosakrale Stenosen

- Malformation der Processus articulares der Wirbel L7 bzw. S1

- Osteochondrosis dissecans (OCD) der kranialen Endplatte des Os sacrum

- akute Bandscheibenvorfälle (HansenTyp-I-Diskusprolaps)

- Diskospondylitis

- traumatische Frakturen, Luxationen oder Subluxationen der Wirbel L7 und/ oder des Os sacrum

- extradurale Zysten

- primäre oder sekundäre Neoplasien (selten)

- Tethered cord syndrome (selten)

- Neuritis (selten)

- spinales Empyem (selten)

\section{DLSS}

Bei der DLSS handelt es sich um eine multifaktorielle Erkrankung, die durch unterschiedliche Kombination einer

- chronischen Diskushernie (HansenTyp-II-Diskushernie) bei L7-S1,

- Hypertrophie von Weichteilstrukturen (Ligamente und synoviale Strukturen),

- Osteophytose der artikulären Facettengelenke, 

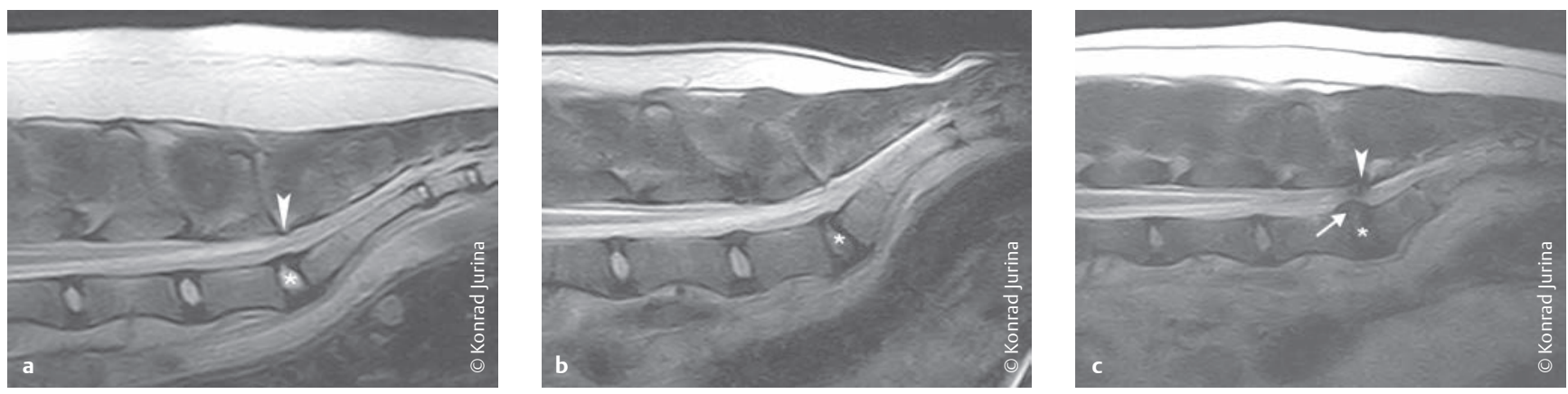

Abb. 1 Kernspintomografie der lumbosakralen Wirbelsäule in sagittaler T2-Wichtung. a Normalbefund: Der normal hydrierte Nucleus pulposus $\left({ }^{*}\right)$ stellt sich hyperintens dar. Der Anulus fibrosus ist ein dünner hypointenser Saum in normaler Position. Das Lig. interarcuale (Pfeilspitze) ist als feine Linie darstellbar. Das epidurale Fettgewebe stellt sich hyperintens im Gegensatz zu den leicht hypointensen Spinalnerven dar. Der Durchmesser des Spinalkanals ist regulär. b Diskusdegeneration: Das Signal des Nucleus pulposus ( $\left.{ }^{*}\right)$ ist hypointens. Andere Veränderungen liegen nicht vor. c Lumbosakrale Stenose: Die degenerierte Bandscheibe $\left(^{*}\right)$ wölbt sich in den Wirbelkanal vor (Pfeil). Das Lig. interarcuale ist hypertrophiert (Pfeilspitze) und trägt zusätzlich zu einer Stenose des Wirbelkanals bei. Die Spinalnerven der Cauda equina sind deutlich komprimiert.

- lumbosakralen Spondylose und

- lumbosakralen Instabilität charakterisiert ist [7].

Weiterhin kommt kongenitalen Veränderungen, insbesondere Übergangswirbeln, eine Bedeutung zu [9].

\section{Pathogenese}

Die Lumbosakralregion weist eine vermehrte Beweglichkeit im Vergleich zu anderen Bereichen der Wirbelsäule auf. Dies ist besonders in Bezug auf die Flexion-Extension, aber auch Torsion oder Biegung ausgeprägt $[2,12]$. Diese vermehrte Beweglichkeit prädisponiert die Hunde für eine Degeneration der Bandscheibe bei L7-S1. Hierdurch kann die auftretende Belastung nicht mehr ausreichend kompensiert werden, was zu einer vermehrten Proliferation der knöchernen Strukturen und umgebenden Weichteilstrukturen führt. Es kommt zu einer Ausbildung von Osteophyten und ventraler bzw. lateraler Spondylose sowie einer Hypertrophie des Lig. interarcuale und Verdickung der Gelenkkapseln der Facettengelenke. Die degenerierte Bandscheibe wölbt sich zunehmend in den Wirbelkanal vor [22].

Das Resultat dieser Veränderungen ist eine Stenose des lumbosakralen Wirbelkanals und evtl. auch eine Einengung der Neuroforaminae ( $\bullet$ Abb.1). Weiterhin ist eine zellvermittelte Entzündung vorhanden, die einen Schmerzreiz induziert.
Klinische Symptome entstehen entweder durch eine direkte Kompression der Cauda equina, das Impingement (Einklemmung) der Spinalnervenwurzeln und Spinalnerven bei Durchtritt durch das Neuroforamen oder eine Kombination daraus [10]. Weiterhin kann auch eine Beeinträchtigung des regionalen Blutflusses eine Rolle spielen [22].

\section{Vorkommen}

Die DLSS tritt vor allem bei adulten Hunden großer Rassen auf. Das Durchschnittsalter bei Vorstellung liegt bei 7 Jahren. Männliche Tiere sind häufiger betroffen. Es besteht eine Prädisposition für den Deutschen Schäferhund [4, 5, 21, 22].

Weder die Heretabilität noch der Vererbungsmodus konnten bisher definiert werden. Es ist am ehesten von einer komplexen multifaktoriellen Pathogenese mit einer polygenen Ursache und dem Einfluss von Umweltfaktoren auszugehen. Neben den genannten Ursachen trägt beim Deutschen Schäferhund das vermehrte Vorkommen von Übergangswirbeln $[9,26]$, eine unterschiedliche Angulierung der Facettengelenke und ein kleinerer Durchmesser des Wirbelkanals im Bereich des Os sacrum im Vergleich mit anderen großen Hunderassen [24] zur Häufung eines Cauda-equina-Syndroms bei.

Eine DLSS ist mittlerweile auch bei Katzen beschrieben [3].

\section{Klinik}

Die Mehrzahl der Hunde mit einem Cauda-equina-Syndrom wird wegen Schmerzen oder Lahmheit einer Hintergliedmaße vorgestellt. Nur selten sind neurologische Ausfälle vorhanden.

Die Besitzer berichten in der Regel von einer reduzierten Aktivität, Problemen beim Aufstehen bzw. Hinsetzen, beim Treppensteigen oder Springen ins Auto. Teilweise zeigen die Hunde dabei Schmerzäußerungen oder sie ziehen eine Hintergliedmaße an. Es kann eine Kyphose mit unter dem Rumpf gestellten Hinterbeinen vorliegen. Die Hunde laufen mit trippelnden Schritten. Oftmals verstärken sich die Symptome unter der Bewegung. Dies ist in einem vermehrten Blutfluss in der lumbosakralen Region begründet, der zu einer verstärkten Kompression der Cauda equina führt.

Bei Beeinträchtigung der Neuroforaminae bzw. der Spinalnervenwurzeln kann auch isoliert eine bis zu hochgradige Lahmheit eines Hinterbeins vorliegen, die evtl. nach Belastung stärker ist. Die Gliedmaße wird im Stehen in starker Flexion entlastet. Man spricht von einem Wurzelirritationssyndrom (Root signature), das durch einen ausstrahlenden Schmerz im Bereich des N. ischiadicus bedingt ist ( $\bullet \mathbf{A b b} .2)$. Mitunter ist ein Zittern der Muskulatur beim Hinsetzen vorhanden.

Die Rute wird vermehrt nach unten gehalten und nicht mehr komplett nach oben geführt. Weiterhin ist eine Schmerzhaftigkeit im lumbosakralen 
Tab. 1 Funktion der peripheren Nerven der Cauda equina und neurologische Befunde bei Hunden mit DLSS (modifiziert nach [22]).

\begin{tabular}{|c|c|c|c|c|}
\hline Nerv & $\begin{array}{l}\text { Ursprung im Rücken- } \\
\text { marksegment }\end{array}$ & Reflex & normale Funktion & neurologische Befunde bei DLSS \\
\hline N. femoralis & L4-L6 & Patellarreflex & $\begin{array}{l}\text { Beugung Hüftgelenk, } \\
\text { Streckung Kniegelenk }\end{array}$ & $\begin{array}{l}\text { normaler Patellarreflex oder Pseudo- } \\
\text { hyperreflexie }\end{array}$ \\
\hline N. ischiadicus & L6-S1 & $\begin{array}{l}\text { Flexorreflex, Tibialis- } \\
\text { cranialis-Reflex, } \\
\text { Gastrocnemius-Reflex }\end{array}$ & $\begin{array}{l}\text { Streckung Hüftgelenk, } \\
\text { Beugung Kniegelenk, } \\
\text { Beugung und Streckung } \\
\text { Tarsalgelenk, Proprio- } \\
\text { zeption }\end{array}$ & $\begin{array}{l}\text { normale oder verminderte Reflexe, } \\
\text { normale oder verminderte Pro- } \\
\text { priozeption, Muskelatrophie und/ } \\
\text { oder verminderter Tonus (vor allem } \\
\text { lange Sitzbeinmuskulatur) }\end{array}$ \\
\hline $\begin{array}{l}\text { N. pelvinus und } \\
\text { Nn. sacrales }\end{array}$ & $\mathrm{S} 1-\mathrm{S} 3$ & & Harnblasenfunktion & kein Befund oder Urininkontinenz \\
\hline N. pudendus & $\mathrm{S} 1-\mathrm{S3}$ & Perinealreflex & Anal- und Blasensphinkter & $\begin{array}{l}\text { kein Befund oder verminderter } \\
\text { Perinealreflex, kein Befund oder Urin- } \\
\text { und Kotinkontinenz }\end{array}$ \\
\hline Nn. caudales & $\mathrm{Cd} 1-\mathrm{Cd} 5$ & & Tonus Rute & normal oder Hypotonie \\
\hline
\end{tabular}

Bereich, selten auch eine Automutilation dieser Region vorhanden.

Bei Hunden, die im Hundesport oder als Diensthunde eingesetzt werden, treten die Symptome während der erhöhten Anforderung im Training oder der Ausbildung auf. Die Leistungsbereitschaft lässt nach. Aus Erfahrung des Autors haben die Tiere oftmals schon umfangreiche orthopädische Abklärungen hinter sich, die keine Ursache identifizieren konnten.

Neurologische Defizite sind selten und meist erst bei fortgeschrittener Erkrankung vorhanden. Dies liegt in erster Linie daran, dass Nerven generell, also auch die der Cauda equina, resistenter gegenüber einer Stenose des Wirbelkanals sind, als beispielsweise das Rückenmark selbst. Es liegen daher bei deutlichen neurologischen Defiziten oftmals andere oder zusätzliche Läsionen vor. Vor allem eine Diskushernie oder ein Diskusprolaps an anderer Lokalisation, eine degenerative Myelopathie oder Neoplasie an anderer Lokalisation sollten ausgeschlossen werden.

Die neurologischen Defizite sind in erster Linie von den beteiligten Nervenwurzeln bzw. Spinalnerven und deren Funktion abhängig ( $\triangleright$ Tab. 1$)$.

Es liegen Veränderungen in der motorischen oder sensorischen Funktion der Hintergliedmaßen, der Rute oder der Perinealregion vor, die auch asymmetrisch ausfallen können. Diese äußern sich als milde Propriozeptionsstörungen, Zehenschleifen, Paresen der Gliedma- ßen, verminderte Beugung des Tarsalgelenks und verminderter Rutentonus. Eine Urin- und/oder Kotinkontinenz kann in seltenen Fällen auftreten.

Diese klinischen Zeichen können alleine oder in den unterschiedlichsten Kombinationen vorkommen. Eine Paraplegie, d.h. ein kompletter Verlust der willkürlichen Motorik der Hintergliedmaßen ist bei einer DLSS nicht vorhanden.

\section{Klinische Untersuchung}

Die klinische Untersuchung sollte eine Allgemeinuntersuchung zum Ausschluss anderer Krankheiten sowie eine komplette neurologische und orthopädische Untersuchung beinhalten.

Dies ist insbesondere wichtig, da im Falle einer Lahmheit die klinischen Symptome oftmals nicht sicher einer Erkrankung des Bewegungsapparats oder des Nervensystems zugeordnet werden können. Weiterhin sind bei Hunden mit einem Cauda-equina-Syndrom auch zahlreiche orthopädische Erkrankungen, wie z.B. eine Hüftgelenksdysplasie oder eine Kniegelenkspathologie als Komorbidität möglich. Diese sollten im Rahmen der Untersuchung ausgeschlossen werden.

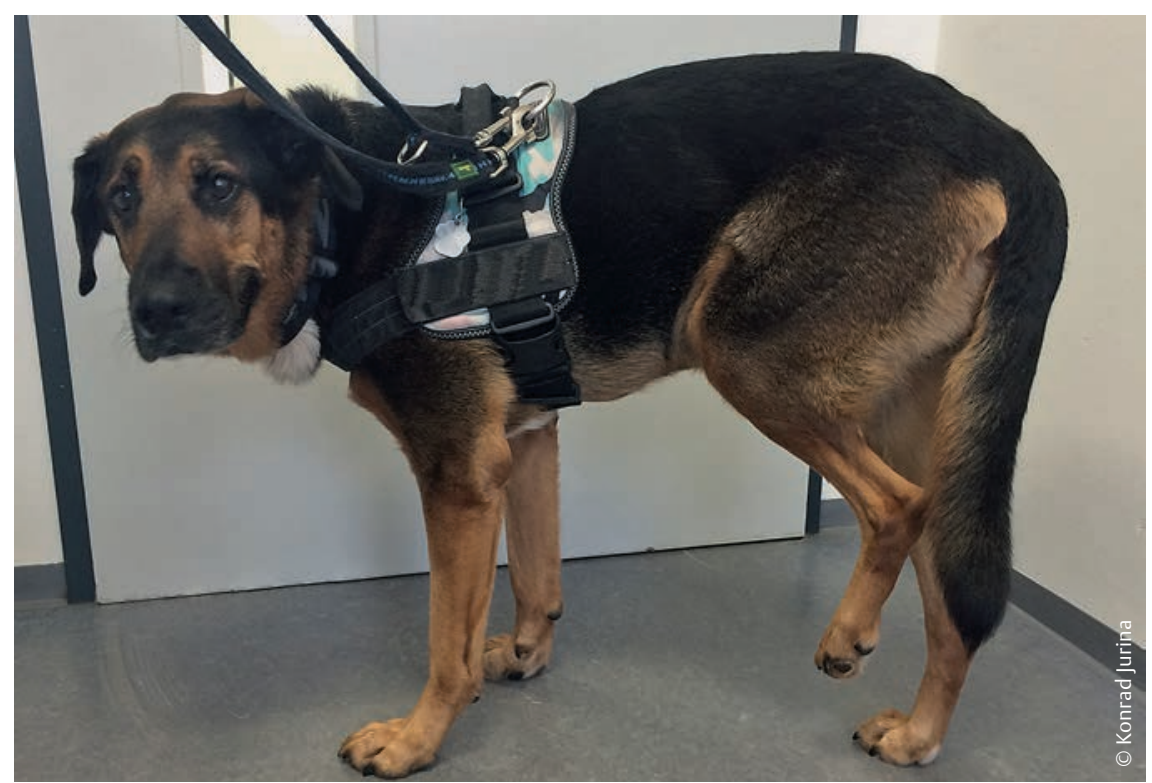

Abb. 2 Mischling, männlich-kastriert, 9 Jahre: Wurzelirritationssyndrom mit hochgradiger Lahmheit der linken Hintergliedmaße und Schmerzen beim Springen wegen einer neuroforaminalen Stenose bei L7-S1. 


\section{Druckpalpationstests}

Im Vordergrund der klinischen Befunde steht eine Dolenz, die durch Druckpalpation der Lumbosakralregion provoziert wird. Die Testauswahl ist abhängig von der Kooperationsbereitschaft des Hundes und der Vorliebe des Untersuchers. In der Regel reicht die Provokation der Dolenz eines Tests aus, um klinisch den Verdacht auf ein Cauda-equina-Syndrom zu äußern.

Folgende Tests können durchgeführt werden:

- Dorsaler Druck im Bereich des Lig. interarcuale von L7-S1 bei gleichzeitiger Hyperextension der lumbalen Wirbelsäule (Lordose-Test). Es sollte darauf geachtet werden, die Hüftgelenke nicht übermäßig zu strecken, um eine dort lokalisierte Schmerzursache auszuschließen ( $\triangleright$ Abb. $3 a$ ).

- Hyperextension der Rute mit oder ohne zusätzlichen lumbosakralen Druck.

- Hyperextension einer einzelnen Hintergliedmaße und bei gleichzeitigem lumbosakralem Druck ( Abb. $\mathbf{3 b}$ ). Hierdurch kann eine Lateralisation der Schmerzen bestätigt werden. In vielen Fällen führt dies zu einer verstärkten Lahmheit bzw. vermehrten Entlastung direkt nach der Untersuchung [10]. Der Besitzer sollte im Vorfeld der Untersuchungen hierüber informiert werden.
- Seitlicher paravertebraler Druck unmittelbar kranial des Os ilium in der Vertiefung der paravertebralen Muskulatur ( Abb. 3c). Der Autor favorisiert bei Verdacht auf ein Wurzelirritationssyndrom diesen Test gegenüber den anderen angeführten. Hier liegt ein Dermatom der Dorsalwurzel des L7 vor, wodurch bei betroffenen Tieren oftmals eine hochgradige Schmerzreaktion hervorgerufen wird.

- Druckpalpation im weiteren Verlauf des N. ischiadicus im Bereich der kaudalen Oberschenkelmuskulatur zwischen den Mm. semitendinosus/semimembranosus und dem M. biceps femoris ( $\triangleright$ Abb. 3d) [7].
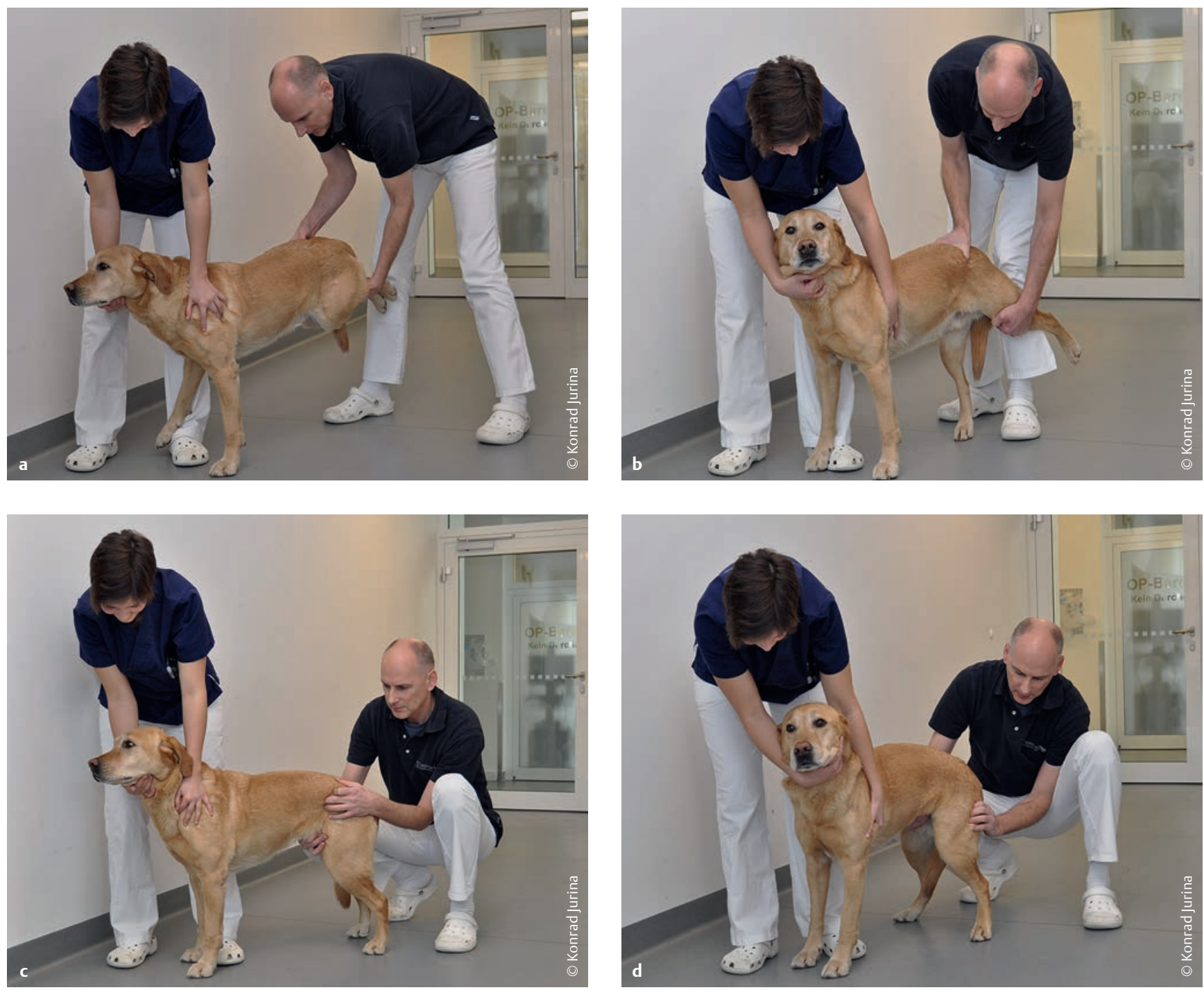

Abb. 3 Methoden der Druckpalpation zur Evaluation einer lumbosakralen Dolenz. a Lordose-Test: Dorsaler Druck im Bereich des Lig. interarcuale von L7-S1 bei gleichzeitiger Hyperextension der lumbalen Wirbelsäule. $\mathbf{b}$ Hyperextension einer einzelnen Hintergliedmaße und bei gleichzeitigem lumbosakralem Druck. c Seitlicher paravertebraler Druck unmittelbar kranial des Os ilium in der Vertiefung der paravertebralen Muskulatur. $\mathbf{d}$ Druckpalpation des N. ischiadicus im Verlauf der kaudalen Oberschenkelmuskulatur. 
- Rektale Palpation des Verlaufs des $\mathrm{N}$. ischiadicus und der ventralen Umgebung der Bandscheibe von L7-S1. Neben einer Dolenz können Raumforderungen in der Beckenhöhle oder Prostataveränderungen erfasst werden.

Es sollte eine beidseits vergleichende Palpation der Oberschenkelmuskulatur vorgenommen werden. Hierbei können oft eine Muskelatrophie und ein verminderter Tonus der langen Sitzbeinmuskulatur bemerkt werden [10].

Neurologische Defizite sind in der Regel Läsionen vom Typ eines unteren motorischen Neurons der Hintergliedmaßen $(\triangleright$ s. Kasten). Die spinalen Reflexe, insbesondere der Flexorreflex, können reduziert sein. Beim Flexorreflex wird oftmals insbesondere die Beugung im Tarsalgelenk nur unvollständig vorgenommen. Meist ist die Beugung im Knie- und Hüftgelenk normal. Im Gegensatz dazu kann der Patellarreflex selten auch erhöht sein. Es handelt sich um eine Pseudo-Hyperreflexie, die durch den erhöhten Tonus der Kniegelenksstrecker (durch den N. femoralis innerviert) und den verminderten Tonus der Gliedmaßenbeuger (durch den N. ischiadicus innerviert) bedingt ist.

Der Anal- und Perinealreflex kann vermindert sein. Bei der rektalen Untersuchung fällt dann zumeist auch ein verminderter Analtonus auf. In Fällen einer Kot- und/oder Urininkontinenz kann der Perineal- und Inguinalbereich entzündet sein.

\section{Diagnostik}

Eine Verdachtsdiagnose wird anhand des Vorberichts und der klinischen Untersuchung gestellt. Um den Verdacht zu bestätigen, sind bildgebende Verfahren notwendig.

Mittlerweile ist dabei die Kernspintomografie (MRT = Magnetresonanztomografie), u.U. auch die Computertomografie (CT) die Methode der Wahl.

Die früher verwendeten röntgenologischen Techniken wie Myelografie, Diskografie und Epidurografie wurden durch die Weiterentwicklung der Schnittbildtechnik und die mittlerweile breite Anwendung in der Tiermedizin ersetzt.

\section{Oberes und unteres motorisches Neuron}

Mit den Begriffen „oberes motorisches Neuron“ (OMN) und „unteres motorisches Neuron“ (UMN) werden funktionell die Anteile des Nervensystems charakterisiert, die für die Motorik verantwortlich sind. Das OMN reguliert das UMN.

Das OMN besteht neuroanatomisch aus den motorischen Kernen im Gehirn sowie den Leitungsbahnen im Rückenmark. Das UMN stellt den efferenten Anteil des spinalen Reflexbogens dar und besteht somit aus den $\alpha$-Motoneuronen in der Ventralwurzel des Rückenmarks, den Spinalnervenwurzeln, Spinalnerven und der neuromuskulären Endplatte.

Klinisch bedeutsam ist die Unterscheidung für die Untersuchung der spinalen Reflexe:

- Bei einer Läsion des UMN ist der Reflexbogen beeinträchtigt. Die spinalen Reflexe sind daher vermindert oder abwesend.

- Bei einer Läsion des OMN ist der Reflexbogen nicht verändert. Die spinalen Reflexe sind daher normal, evtl. sogar gesteigert.

\section{Nativröntgen}

Nativröntgenaufnahmen können für eine erste Übersicht und zum Ausschluss von Differenzialdiagnosen wie z.B. osteolytischen Neoplasien, Traumata oder einer fortgeschrittenen Diskospondylitis eingesetzt werden. Die Aufnahmen sollten vorzugsweise in Narkose/ Sedation erfolgen, um gut gelagerte, überlagerungsfreie Bilder des lumbosakralen Übergangs in 1/1- und v/d-Projektion zu erhalten.

Ein weiterer Vorteil der Röntgenaufnahmen liegt in der Möglichkeit, Aufnahmen in Extension und Flexion der Wirbelsäule anzufertigen. Hierbei kann eine mögliche lumbosakrale Instabilität mit Wirbelgleiten erkannt werden [18].
Hinweise für eine DLSS auf Nativröntgenaufnahmen können sein:

- eine Reduktion bzw. ein Kollaps des Intervertebralspalts von L7-S1

- eine Sklerose der Wirbelendplatten

- die Ausbildung ventraler und/oder lateraler Spondylosen

- eine ventrale Subluxation des Os sacrum in Relation zum Wirbelkörper von L7

- eine Verlängerung der dorsalen Lamina des Os sacrum unter die kaudale Begrenzung von L7 ( $\triangleright$ Abb. 4)

Weiterhin können eine OCD-Läsion am kraniodorsalen Rand des Os sacrum [11], eine Gasansammlung im Intervertebralspalt (Vakuumphänomen) sowie Über-

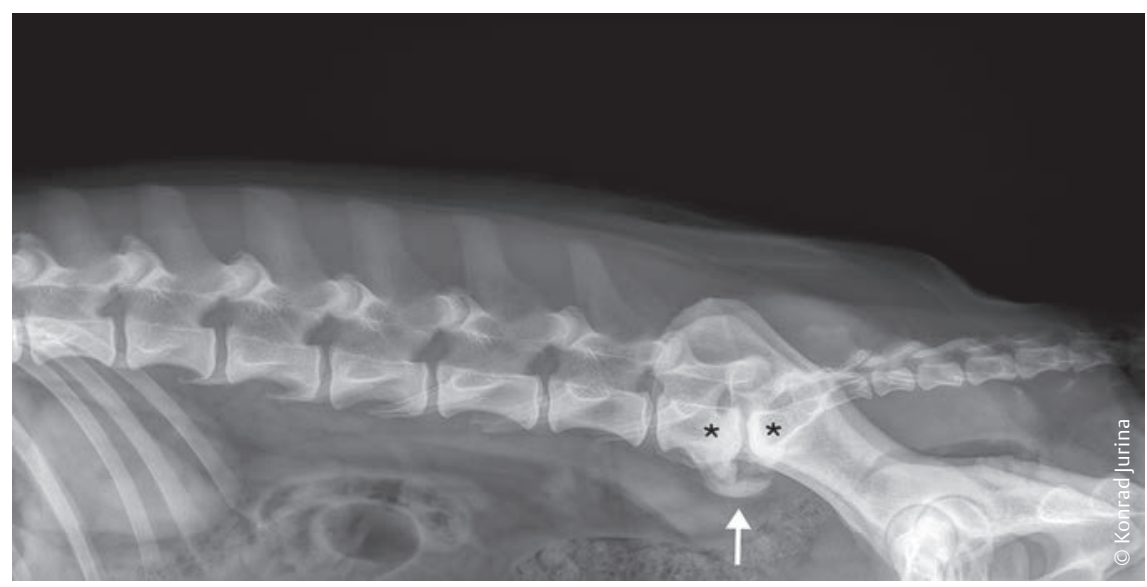

Abb. 4 DSH, männlich, 7 Jahre; Röntgenaufnahme in laterolateraler Projektion. Der Hund zeigte Schmerzen im Hundesport. Es sind als Zeichen einer DLSS eine Verengung des Intervertebralspalts, eine ventrale Spondylose (Pfeil) sowie eine Sklerosierung der Wirbelendplatten von L7 bzw. S1 ( ${ }^{*}$ ) sichtbar. Das kraniale Dach des Os sacrum reicht „teleskopartig“ unter die kaudale Lamina des L7. 
gangswirbel und knöcherne Malformationen identifiziert werden [28].

Bei den Röntgenbildern schließt ein unauffälliger Befund eine lumbosakrale Problematik nicht aus. Ebenso sind aber auch hochgradige degenerative Veränderungen, die röntgenologisch sichtbar sind, nicht beweisend für eine DLSS [25].

\section{CT und MRT}

Der Vorteil der beiden Schnittbildverfahren besteht in der überlagerungsfreien Darstellung der relevanten Strukturen des lumbosakralen Übergangs. Im Gegensatz zur CT kann die MRT besser die Weichteilveränderungen darstellen. Beide Methoden haben das Verständnis der pathologischen Veränderungen und vor allem die chirurgischen Therapieoptionen revolutioniert.

Es können vor allem in transversaler Schnittrichtung Querschnitte angefertigt werden, wodurch der Verlauf der Spinalnervenwurzeln und speziell die Neuroforaminae beurteilt werden können. Wichtig sind dabei geeignete Parameter der verwendeten Protokolle. Insbesondere die Schnittdicke der einzelnen Schichten sollte unterhalb von $1 \mathrm{~mm}$ liegen.

Eine Fragestellung, deren Bedeutung noch nicht abschließend geklärt ist, betrifft eine mögliche lumbosakrale Instabilität. Mittels der Schnittbildgebung können ebenfalls dynamische Untersuchungen in Flexion und Extension vorgenommen werden und somit ein Wirbelgleiten oder eine positionsabhängige Verstärkung der Befunde identifiziert werden.

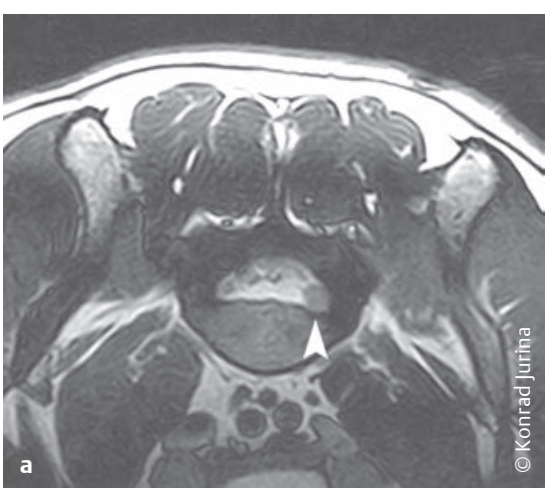

CT

Die Befunde der CT sind analog zum Röntgen. Zusätzlich können aber eine Diskushernie, eine Hypertrophie des Lig. interarcuale, eine Verdickung der Gelenkkapsel und eine foraminale Stenose erkannt werden. Kalzifizierungen und ein Vakuumphänomen können sicher identifiziert werden. Eine OCD-Läsion ist mitunter eindeutiger zu identifizieren.

\section{MRT}

Die MRT bietet detaillierte Informationen zur Degeneration der Bandscheibe, zum Duraschlauch und vor allem in Bezug auf die foraminale Stenose und deren Auswirkungen auf die Spinalnervenwurzeln. Infolge des chronischen Druckes kommt es zu einer kompensatorischen Querschnittsvergrößerung ( $\triangleright$ Abb. 5), vor allem der dorsalen Spinalnervenwurzel mit Einlagerung von „Polsterkissen“, sogenannten Renaut-Körperchen. Dies kann in der MRT-Bildgebung als eine deutliche Signaländerung dargestellt werden [19].

Auch die Beurteilung des Neuroforamens, vor allem die Lokalisation einer Stenose, kann sicher identifiziert werden. Eine Vielzahl von Patienten mit einer DLSS, bei denen die Lahmheit einer Hintergliedmaße das vorherrschende klinische Bild ist, hat eine Stenose der Austrittszone des Neuroforamens durch bindegewebige Zubildungen [10]. In einigen Fällen kann diese auch bilateral vorhanden sein. Mittels der MRT kann daher eine eventuelle Operation detaillierter geplant werden.

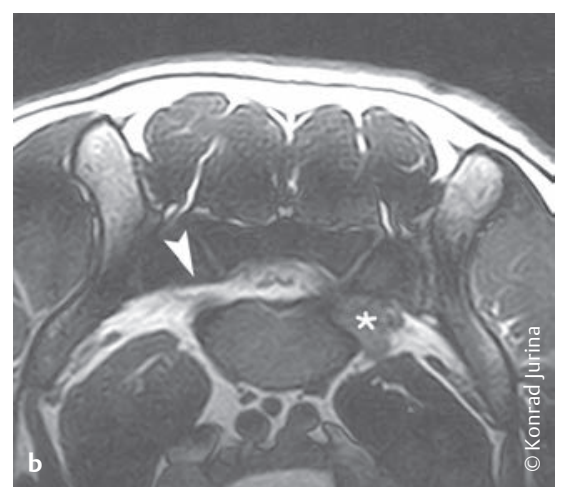

Abb. 5 DSH, männlich, 5 Jahre; hochgradige Lahmheit der linken Hintergliedmaße. Kernspintomografie in 3D-Gradientenwichtung (3DHYCE) in transversaler Schnittrichtung. a Es ist eine deutliche Verdickung (Pfeilspitze) der dorsalen und ventralen Spinalnervenwurzeln von L7 im kranialen Bereich des Neuroforamens bzw. b eine hochgradige Stenosierung der Austrittszone im weiter kaudalen Verlauf des Neuroforamens durch leicht hyperintenses Material ( $\left.{ }^{*}\right)$ nachweisbar. Das rechte Neuroforamen (Pfeilspitze) stellt sich unauffällig dar.
$\mathrm{Zu}$ beachten ist bei der Bildgebung, dass auch andere Läsionen im Bereich der Wirbelsäule an anderer Lokalisation vorliegen können. Gerade bei Patienten mit neurologischen Ausfällen ist es daher wichtig, die gesamte Wirbelsäule zu untersuchen, um nicht weitere Pathologien zu übersehen.

\section{Bedeutung der Befunde}

Insgesamt besteht eine hohe Korrelation zwischen den Befunden der CT und MRT, aber eine geringe im Hinblick auf die Bildgebung und den intraoperativen Befund $[16,29]$. Dies mag teilweise durch eine unterschiedliche Lagerung der Hunde erklärt sein.

Ein Problem der bildgebenden Verfahren ist daher nach wie vor, dass es nicht möglich ist, eine genaue Abgrenzung zwischen Veränderungen, die für das klinische Bild verantwortlich sind, und solchen, die einen Zufallsbefund darstellen, zu treffen. Weiterhin gibt es keine Korrelation zwischen der Schwere der Befunde der bildgebenden Diagnostik und der klinischen Symptomatik $[1,15,20]$. Möglicherweise können unterstützende Verfahren wie eine Elektrodiagnostik oder eine genauere Bewegungsanalyse mittels „Force plate“ zukünftig dieses Dilemma lösen [21, 27].

\section{Differenzialdiagnosen}

Mögliche Differenzialdiagnosen sind in - Tab. 2 dargestellt. Die meisten, gerade orthopädische Beschwerden, können durch eine sorgfältige klinische Untersuchung ausgeschlossen werden. Zu beachten ist allerdings, dass ältere Hunde andere degenerative Erkrankungen zusätzlich zu lumbosakralen Veränderungen aufweisen können.

\section{Therapie}

Wie bei den meisten bandscheiben-assoziierten Erkrankungen kann eine konservative/medikamentelle oder eine chirurgische Therapie vorgenommen werden. Die Entscheidung richtet sich in der Regel nach dem Schweregrad der klinischen Befunde, etwaigen Begleiterkrankungen, dem Alter, aber auch der Nutzung des Hundes, den Möglichkeiten des Besitzers und weiteren Faktoren. 


\section{Konservative/medikamentelle Therapie}

In vielen Fällen wird, auch ohne bildgebende Diagnostik, zuerst eine medikamentelle Therapie vorgenommen. Dies gilt besonders für Patienten mit lumbosakralen Schmerzen.

Auch wenn durch eine symptomatische Therapie die Grundursache der Dolenz nicht beseitigt wird, ja meist eine definitive Diagnose nicht feststeht, ist die Gefahr einer irreversiblen Schädigung der Nerven der Cauda equina in der Regel nicht vorhanden. Der Autor beginnt in den meisten Fällen mit einer medikamentellen Therapie, deren Erfolg aber engmaschig kontrolliert werden muss, um eine Rezidivierung oder Verschlechterung frühzeitig zu erkennen.

Ein integraler Therapieansatz besteht in einer Ruhighaltung über einige Wochen mit Leinenzwang, Vermeidung aller Belastungen, die besonders die lumbosakrale Region betreffen (z.B. Hundesport, extensives Spielen mit anderen Hunden, Treppensteigen, Springen ins Auto, ausgedehnte Wanderungen in den Bergen) sowie einer Gewichtsreduktion bei übergewichtigen Patienten.
Eine unterstützende physiotherapeutische Behandlung wird unbedingt empfohlen. Hierbei können entspannende Massagen, Lockerungsübungen und weitere Verfahren je nach Indikation angewandt werden.

\section{Medikamente}

Medikamentell kommen Schmerzmedikamente zum Einsatz. Neben der Verabreichung eines nichtsteroidalen Antiphlogistikums (NSAID) sollte vor allem der neuropathische Schmerz adressiert werden. Hierfür wird Gabapentin oder Pregabalin eingesetzt, wobei humanmedizinische Präparate umgewidmet werden müssen. Weiterhin treten schmerzhafte Verspannungen der paravertebralen Muskulatur auf, die mit einem Muskelrelaxans behandelt werden.

Die systemische Gabe eines Kortikoids ist umstritten und sollte wegen potenzieller Nebenwirkungen eher vermieden werden. Während beim Menschen die epidurale oder paravertebrale Kortisoninjektion sehr verbreitet ist, gibt es hierzu nur eine neuere Publikation bei Hunden mit DLSS. Es wurde eine wieder-

Tab. 2 Differenzialdiagnosen zum Cauda-equina-Syndrom des Hundes.

\begin{tabular}{|ll}
\hline Symptome & Differenzialdiagnose \\
\hline Schmerzen & Hüftgelenksdysplasie \\
& partielle oder komplette Kreuzbandruptur \\
& Grazilis- und Semitendinosus-Kontraktur \\
primäre oder sekundäre Neoplasie & \\
& Trauma \\
& Meningitis/Meningomyelitis \\
& Diskospondylitis \\
& Prostatitis \\
& Diskopathie kaudale Lendenwirbelsäule oder Schwanzwirbel \\
& extramedulläre spinale Zysten \\
& kongenitale Malformationen der Wirbel \\
& kongenitale Malformationen der Cauda equina („Tethered cord \\
& syndrome“, Meningozele/Meningomyelozele) \\
& Dermoidsinus \\
& degenerative Myelopathie \\
& thorakolumbale Diskopathie \\
& primäre oder sekundäre Neoplasie \\
& Meningitis/Meningomyelitis \\
& schwere Diskospondylitis \\
& Trauma \\
kongenitale Malformationen der Wirbel \\
kongenitale Malformationen der Cauda equina („Tethered cord \\
syndrome“, Meningozele/Meningomyelozele) \\
Faserknorpelembolie \\
Polyneuropathie \\
arterielle Thrombembolie \\
neurologische Defizite \\
\hline
\end{tabular}

holte epidurale Verabreichung von Methylprednisolonazetat als erfolgreiche Schmerzbehandlung beschrieben [13]. Eine paravertebrale Depot-Kortisoninjektion unter Durchleuchtungskontrolle an die Austrittszone des Neuroforamens wird ebenfalls angewandt.

Die vom Autor favorisierte medikamentelle Therapie stellt $\triangleright$ Tab. 3 dar.

\section{Alternative Verfahren}

Als komplementäre Maßnahme kann auch eine Schmerzbehandlung mittels Akupunktur versucht werden.

\section{Therapieerfolg}

Die konservative/medikamentelle Therapie kann die Grundursache der DLSS nicht beseitigen. In einzelnen Fällen kann aber eine ausreichende Schmerzunterdrückung gelingen, um eine normale Bewegung der Hunde zu erzielen. Eine aktuelle Publikation weist einen Therapieerfolg bei konservativer Behandlung bei $55 \%$ der untersuchten Fälle nach [4]. Je stärker die neurologischen Ausfälle waren, umso geringer war der Therapieerfolg. Dies traf besonders für Tiere mit Kot- und Urininkontinenz zu, bei denen es praktisch zu keiner Besserung kam. Bei vielen Fällen, die konservativ behandelt wurden, besteht aber die Gefahr der Rezidivierung bei stärkerer Belastung der Hunde, auch wenn die Symptome zunächst nicht mehr vorhanden waren.

\section{Chirurgische Therapie}

Eine chirurgische Therapie sollte bei den Patienten eingesetzt werden, bei denen neurologische Defizite vorliegen oder die deutliche, auf eine konservative Behandlung nicht ansprechende lumbosakrale Schmerzen haben. Das Ziel besteht in einer Dekompression der Nerven der Cauda equina und/oder der Druckentlastung eingeklemmter Nervenwurzeln. Es gibt zahlreiche Operationsverfahren, die zur Therapie der DLSS beim Hund beschrieben sind. Eine genaue Darstellung würde den Umfang dieses Artikels sprengen. Es wird auf die entsprechende chirurgische Literatur verwiesen. Die Auswahl und „Dosis“ der Operationsmethode ist entscheidend von den Befunden der Bildgebung abhängig. Eine Rolle spielt dabei, ob lediglich eine 
mediane Diskushernie mit Stenose des Wirbelkanals oder auch zusätzlich oder isoliert eine neuroforaminale Problematik besteht. Es gibt aktuell keine belastbaren evidenzbasierten Studien, ob neben einer Dekompression auch eine Stabilisierung des lumbosakralen Segments oder Distraktion mit Stabilisierung bei der chirurgischen Therapie der DLSS beim Hund vorteilhaft ist.

\section{Dorsale Laminektomie}

Die hauptsächlich angewandte Methode zur Dekompression ist eine dorsale Laminektomie, die zunehmend, um eine $\mathrm{zu}$ große Destabilisierung des lumbosakralen Übergangs zu verhindern, eher als „Mini-Laminektomie“" ausgeführt wird. Bei der klassischen dorsalen Laminektomie wird in der Regel die dorsale Lamina ab der Hälfte von L7, u.U. auch noch weiter nach kranial, sowie die dorsale Lamina von S1 entfernt. Im Gegensatz hierzu beschränkt sich die „Mini-Laminektomie“ auf die Entfernung der Lamina auf S1.

Kombiniert wird die Dekompression oftmals mit einer partiellen Resektion der sich vorwölbenden Bandscheibe von L7-S1 und einer Resektion des hypertrophierten Lig. interarcuale.

\section{Foraminotomie}

Eine Foraminotomie kommt bei lateral gelegenen Stenosen zum Einsatz. Diese hat den Vorteil, ohne eine relevante Destabilisierung zu bewirken, vor allem die Austrittszone des Neuroforamens zu erreichen und somit die Spinalnervenwurzeln wieder freizulegen [10]. Somit können Komplikationen, wie sie bei der früher verbreiteteren Facettektomie aufgetreten sind, verhindert werden.

\section{Stabilisation}

Bei offensichtlicher Instabilität wird zusätzlich eine stabilisierende Operation vorgenommen. Dabei wird mittels verschiedener Implantate eine feste Verbindung zwischen den Wirbelkörpern von L7 und S1 angestrebt.

\section{„Minimal-invasive“-Technik}

Es wurde auch eine „Minimal-invasive“Technik beschrieben, bei der mittels eines transilialen Pins die lumbosakrale Wirbelsäule in maximaler Flexion fixiert wird, und somit die Neuroforaminae geweitet und eine potenzielle Diskushernie reduziert werden [23]. Belastbare Langzeitergebnisse fehlen allerdings bei dieser Methode.

\section{Postoperative Nachsorge}

Im Anschluss an eine chirurgische Dekompression werden ebenfalls eine analgetische Behandlung sowie eine unterstützende Physiotherapie vorgenommen. Die Hunde müssen über einige Wochen strikt ruhig gehalten werden und dürfen erst langsam wieder belastet werden. Eine enge Zusammenarbeit zwischen Physiotherapeuten und Chirurgen ist vorteilhaft. Je nach Operationsmethode ist ein Kurz- und Langzeiterfolg zwischen 67-95\% beschrieben. Auch hier gilt, je stärker die neurologischen Defizite sind, umso schlechter ist die Prognose. Auch eine Kot- und Urininkontinenz, vor allem länger bestehend, ist meist nicht reversibel.

\section{Schlussbemerkung}

Bei der DLSS des Hundes liegen zahlreiche Analogien zu gleichartigen Befunden beim Menschen vor. Obwohl die Krankheit beim Hund bereits 1978 erstmalig beschrieben wurde und sich in den letzten Jahren zahlreiche Veröffentlichungen mit der DLSS beim Hund beschäftigt haben, gibt es noch viele offene Fragen. Dies liegt in erster Linie an der fehlenden Vergleichbarkeit der vorgenommenen Untersuchungen.

Zweifelsfrei hat die breitflächige Anwendung der modernen Schnittbildverfahren wie CT oder MRT und kinematischer Studien zu einem besseren Verständnis der Veränderungen geführt. Hierdurch konnten insbesondere chirurgische Verfahren optimiert werden. Es gibt aber keine belastbaren Informationen, welche Therapiemethode bei welchen klinischen Symptomen oder Befunden der

Tab. 3 Wirkstoffe und Dosierung zur medikamentellen Therapie lumbosakraler Schmerzen.

\begin{tabular}{|c|c|c|c|c|}
\hline Komponenten & Wirkstoff & Dosis (mg/kg KM) & Wirkdauer & Verabreichung \\
\hline \multirow[t]{7}{*}{ NSAID } & Carprofen & 4 & $24 \mathrm{~h}$ & p.o., s.c., i.v. \\
\hline & Cimicoxib & 2 & $24 \mathrm{~h}$ & p.o. \\
\hline & Firocoxib & 5 & $12-24 \mathrm{~h}$ & p.o. \\
\hline & Meloxicam & 0,2 am 1. Tag, dann 0,1 ab 2. Tag & $24 \mathrm{~h}$ & p.o., s.c., i.m. \\
\hline & Robenacoxib & $1-2$ & $24 \mathrm{~h}$ & p.o., s.c. \\
\hline & Tepoxalin & 10 & $24 \mathrm{~h}$ & p.o. \\
\hline & Metamizol & $20(-50)$ & $6-8 h$ & p.o., s.c. \\
\hline NSAID + Prednisolon* & $\begin{array}{l}\text { Phenylbutazon } 50 \mathrm{mg}+ \\
\text { Prednisolon } 1,5 \mathrm{mg}^{*}\end{array}$ & 1 Tbl. pro 15 kg KM & $12 \mathrm{~h}$ & p.o. \\
\hline Muskelrelaxans & Metocarbamol** & $25-30$ & $8 \mathrm{~h}$ & p.o. \\
\hline \multirow[t]{2}{*}{ neuropathischer Schmerz } & Gabapentin** & $7-10$ & $8 \mathrm{~h}$ & p.o. \\
\hline & Pregabalin** & $2(-4)$ & $12 \mathrm{~h}$ & p.o. \\
\hline
\end{tabular}

* = als 2. Option, wenn NSAID nicht ausreichend wirksam sind, ${ }^{* *}=$ Umwidmung aus Humanmedizin 
Bildgebung die am besten geeignete wäre. Ein bisher nicht gelöstes Dilemma ist, sicher beurteilen zu können, ob die in der Bildgebung erhobenen Befunde tatsächlich mit den klinischen Symptomen korrelieren.

Es bleibt daher bisher beim Appell, entsprechende Studien mit robusten Einschlusskriterien, objektiven und definierten Nachkontrollen und idealerweise randomisierten Therapiestudien vorzunehmen [14].

Online zu finden unter

http://dx.doi.org/10.1055/s-0042-102304

\section{Literatur}

1 Axlund TW, Hudson JA. Computed tomography of the normal lumbosacral intervertebral disc in 22 dogs. Vet Radiol Ultrasound 2003; 44 (6): 630-634

2 Benninger MI, Seiler GS, Robinson LE et al. Effects of Anatomic Conformation on Three-Dimensional Motion of the Caudal Lumbar and Lumbosacral Portions of the Vertebral Column of Dogs. Am J Vet Res 2006; 67: 43-50

3 Danielski A, Bertran J, Fitzpatrick N. Management of Degenerative Lumbosacral Disease in Cats by Dorsal Laminectomy and Lumbosacral Stabilization. Vet Comp Orthop Traumatol 2013; 26 (1): 69-75

4 De Decker S, Wawrzenski LA, Volk HA. Clinical Signs and Outcome of Dogs Treated Medically for Degenerative Lumbosacral Stenosis: 98 Cases (2004-2012). J Am Vet Med Assoc 2014; 245: 408-413

5 De Risio L, Sharp NJH, Olby NJ et al. Predictors of Outcome After Dorsal Decompressive Laminectomy for Degenerative Lumbosacral Stenosis in Dogs: 69 Cases (1987-1997). J Am Vet Med Assoc 2001; 219 (5): 624-628

6 Evans HE, de Lahunta A. Miller's Anatomy of the Dog, 4. Ausg. St. Louis Missouri: Elsevier; 2013

7 Farrel F, Fitzpatrick N. Lumbosacral Disc Disease: Is Vertebral Stabilization Indicated? In: Fingeroth JM, Thomas WB (Hrsg.). Advances in Intervertebral Disc Disease in Dogs and Cats. Ames, lowa: Wiley Blackwell; 2015: 237-250
8 Fletcher TF, Kitchell RL. Anatomical studies on the spinal cord segments of the dog. Am J Vet Res 1966; 27: 1759-1767

9 Flückiger MA, Damur-Duric N, Hässig M at al. A Lumbosacral Transitional Vertebra in the Dog Predisposes to Cauda Equina Syndrome. Vet Radiol Ultrasound 2006; 47 (1): 39-44

10 Goedde T, Steffen F. Surgical Treatment of Lumbosacral Foraminal Stenosis Using a Lateral Approach in Twenty Dogs with Degenerative Lumbosacral Stenosis. Vet Surg 2007; 36 (7): 705-713

11 Hanna FY. Lumbosacral osteochondrosis: radiological features and surgical management in 34 dogs. J Small Anim Pract 2001; 42: 272-278

12 Hediger KU, Ferguson SJ, Gedet P et al. Biomechanical Analysis of Torsion and Shear Forces in Lumbar and Lumbosacral Spine Segments of Nonchondrodystrophic Dogs. Vet Surg 2009; 38 (7): 874-880

13 Janssens L, Béosier Y, Daems R. Lumbosacral Degenerative Stenosis in the Dog. The Results of Epidural Infiltration with Methylprednisolone Acetate: A Retrospective Study. Vet Comp Orthop Traumatol 2009; 22: 486-491

14 Jeffery ND, Barker A, Harcourt-Brown TR. What Progress Has Been Made in the Understanding and Treatment of Degenerative Lumbosacral Stenosis in Dogs During the Past 30 Years?. Vet J 2014; 201 (1): 9-14

15 Jones JC, Inzana KD. Subclinical CT Abnormalities in the Lumbosacral Spine of Older Large-Breed Dogs. Vet Radiol Ultrasound 2000; 41 (1): 19-26

16 Jones JC, Banfield CM, Ward DL. Association Between Postoperative Outcome and Results of Magnetic Resonance Imaging and Computed Tomography in Working Dogs with Degenerative Lumbosacral Stenosis. J Am Vet Med Assoc 2000; 216: 1769-1774

17 Lang J. Flexion-Extension myelography of the canine cauda equina. Vet Radiol Ultrasound 1988; 29: 242-257

18 Lang J, Jaggy A. X-ray studies of the cauda equina of dogs. Schweiz Archiv Tierheilk 1989; 131: 299-309

19 Matiasek K, Steffen F, Schmahl W et al. Back to the roots - radicular pathology in foraminal stenosis (5 cases). J Vet Intern Med 2008; 22 (29): 504

20 Mayhew PD, Kapatkin AS, Wortmann JA et al. Association of cauda equina compression on magnetic resonance images and clinical signs in dogs with degenerative lumbosacral stenosis. J Am Anim Hosp Assoc 2002; 38: 555-562
21 Meij BP, Suwankong N, Van den Brom WE et al. Tibial Nerve Somatosensory Evoked Potentials in Dogs with Degenerative Lumbosacral Stenosis. Vet Surg 2006; 35 (2): 168-175

22 Meij BP, Bergknut N. Degenerative Lumbosacral Stenosis in Dogs. Vet Clin North Am Small Anim Pract 2010; 40 (5): 983-1009

23 Müller F. Minimalinvasive Transiliale Vertebralverblockung (MTV) des siebten Lendenwirbels nach degenerativer Lumbosakralstenose (DLSS) beim Hund. Kleintierpraxis 2008; 53 (3): 141-148

24 Ondreka N, Amort KH, Stock KF et al. Skeletal Morphology and Morphometry of the Lumbosacral Junction in German Shepherd Dogs and an Evaluation of the Possible Genetic Basis for Radiographic Findings. Vet J 2013; 196 (1): 64-70

25 Scharf G, Steffen F, Grünenfelder F et al. The Lumbosacral Junction in Working German Shepherd Dogs - Neurological and Radiological Evaluation. J Vet Med Res 2004; 51: 27-32

26 Steffen F, Berger M, Morgan JP. Asymmetrical, Transitional, Lumbosacral Vertebral Segments in Six Dogs: a Characteristic Spinal Syndrome. J Am Anim Hosp Assoc 2004; 40: 328-344

27 Suwankong N, Meij BP, Van Klaveren NJ et al. Assessment of Decompressive Surgery in Dogs with Degenerative Lumbosacral Stenosis Using Force Plate Analysis and Questionnaires. Vet Surg 2007; 36 (5): 423-431

28 Suwankong N, Meij BP, Voorhout G et al. Review and Retrospective Analysis of Degenerative Lumbosacral Stenosis in 156 Dogs Treated by Dorsal Laminectomy. Vet Comp Orthop Traumatol 2008; 21: 285-293

29 Suwankong N, Voorhout G, Hazewinkel HAW et al. Agreement Between Computed Tomography, Magnetic Resonance Imaging, and Surgical Findings in Dogs with Degenerative Lumbosacral Stenosis. J Am Vet Med Assoc 2006; 229: 1924-1929

30 Uemura EE. Fundamentals of Canine Neuroanatomy and Neurophysiology. Ames, lowa: Wiley Blackwell; 2015

\section{Dr. Konrad Jurina \\ Tierklinik Haar \\ Keferloher Straße 25 \\ 85540 Haar \\ jurina@tierklinik-haar.de \\ www.tierklinik-haar.de}




\section{Fragebogen}

\section{Frage 1}

Die häufigste Ursache für ein Cauda-equina-Syndrom beim Hund ist:

a. ein Hansen-Typ-I-Diskusprolaps

b. eine degenerative lumbosakrale Stenose

c. eine Neoplasie

d. eine Fraktur

e. eine entzündliche Läsion

\section{Frage 2}

\section{Eine degenerative lumbosakrale} Stenose (DLSS) ist:

a. häufig bei sehr jungen Hunden

b. häufig bei chondrodystrophen Rassen

c. häufig bei kleinen Hunderassen

d. eine multifaktorielle Erkrankung

e. eine Folge wiederholter Traumata

\section{Frage 3}

Die DLSS tritt vor allem auf bei:
a. Dobermann Pinschern
b. Rottweilern
c. Cockerspaniel
d. Deutschen Schäferhunden
e. Rassekatzen

\section{Frage 4}

Ein Wurzelirritationssyndrom (,Root signature“) äußert sich typischerweise als:
a. vermindertes Kauen auf einer Seite
b. Parese einer Gliedmaße
c. Lahmheit einer Gliedmaße
d. generalisierter Juckreiz
e. Kopfschiefhaltung zu einer Seite

\section{Frage 5}

Welche der folgenden Aussagen ist falsch?

a. Der Patellarreflex wird über den $\mathrm{N}$. femoralis vermittelt.

b. Der Flexorreflex der Hintergliedmaße wird über den $\mathrm{N}$. ischiadicus vermittelt.

c. Der Perinealreflex wird über den $\mathrm{N}$. pudendus vermittelt.

d. Der Ursprung des Patellarreflexes liegt in den Rückenmarkssegmenten S1-S3.

e. Der Ursprung des Flexorreflexes liegt in den Rückenmarkssegmenten L6-S1.

\section{Frage 6}

Für die Diagnostik einer DLSS gilt als Methode der Wahl:

a. eine Laboruntersuchung

b. eine Elektrodiagnostik

c. eine Röntgenuntersuchung

d. eine Szintigrafie

e. eine Schnittbilddiagnostik (CT/MRT)

\section{Frage 7}

Auf Röntgenaufnahmen ...

a. kann eine DLSS zweifelsfrei identifiziert werden.

b. kann eine DLLS zweifelsfrei ausgeschlossen werden.

c. können Diskushernien zweifelsfrei identifiziert werden.

d. können indirekte Hinweise auf eine mögliche DLSS identifiziert werden.

e. kann ein Diskusprolaps zweifelsfrei identifiziert werden.
Frage 8

Mithilfe der Schnittbilddiagnostik (CT/MRT) kann:

a. eine DLSS zweifelsfrei identifiziert werden und eine Korrelation mit den klinischen Befunden bestätigt werden.

b. eine DLSS zweifelsfrei identifiziert werden und eine Korrelation zu den radiologischen Befunden bestätigt werden.

c. eine DLSS nicht immer zweifelsfrei diagnostiziert werden.

d. eine lumbosakrale Instabilität zweifelsfrei diagnostiziert werden. e. eine DLSS identifiziert werden.

\section{Frage 9}

Differenzialdiagnosen zu lumbosakralen Schmerzen beim Caudaequina-Syndrom sind:

a. ein thorakolumbaler Diskusprolaps

b. eine Diskospondylitis

c. eine Myopathie des M. gastrocnemius

d. eine Zystitis

e. ein Analbeutelabszess

\section{Frage 10}

Eine medikamentelle Therapie des neuropathischen Schmerzes erfolgt

z. B. mit:

a. Gabapentin

b. Meloxicam

c. Carprofen

d. Metocarbamol

e. Metamizol 


\title{
Das Cauda-equina-Syndrom des Hundes
}

\author{
Konrad Jurina
}

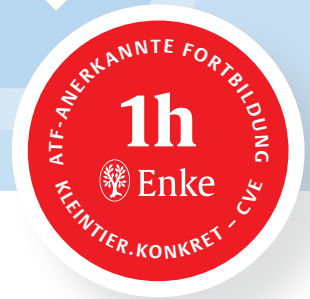

A Lernerfolgskontrolle

\section{Bitte kreuzen Sie die richtigen Antworten an! Es ist jeweils nur 1 Antwort pro Frage richtig!}

\section{B Teilnehmer}

Titel | Name | Vorname

Straße | Hausnummer

PLZ | Ort

Beruf

\section{Ihr Ergebnis wird vom Verlag ausgefültt}

Sie haben von $\square \quad$ Fragen bestanden und 1 ATF-Stunde erhalten.

Stuttgart, den

\section{Teilnahmebedingungen für Abonnenten der kleintier konkret kostenlos}

\section{E Erklärung}

Ich versichere, dass ich die Beantwortung der Fragen selbst und ohne Hilfe durchgeführt habe.

Für diese Fortbildungseinheit können Sie 1 ATFFortbildungsstunde anerkannt bekommen. Hierfür

- müssen mindestens $70 \%$ der Fragen richtig beantwortet sein.

muss der Antwortbogen vollständig ausgefüllt sein. Unvollständig ausgefüllte Bögen können nicht berücksichtigt werden!

Ort, Datum
- muss im markierten Feld* Ihre Abonnentennummer eingetragen oder eine kleintier. konkret-CVE-Wertmarke aufgeklebt sein.

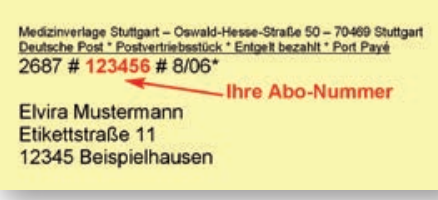

kleintier.konkret-CVE-Wertmarken für NichtAbonnenten können beim Verlag zu folgenden Bedingungen erworben werden: 6er-PackWertmarken, Preis 49,95€ inkl. MWSt., Artikel-Nr. 903000.

Bitte richten Sie die Bestellungen an: Georg Thieme Verlag Stuttgart,

KundenServiceCenter Buch, Postfach 3011 20, 70451 Stuttgart.

* Nicht-Abonnenten bitte hier kleintier. konkret-CVE-Wertmarke aufkleben, Abonnenten bitte Abonnentennummer eintragen.

Unterschrift 